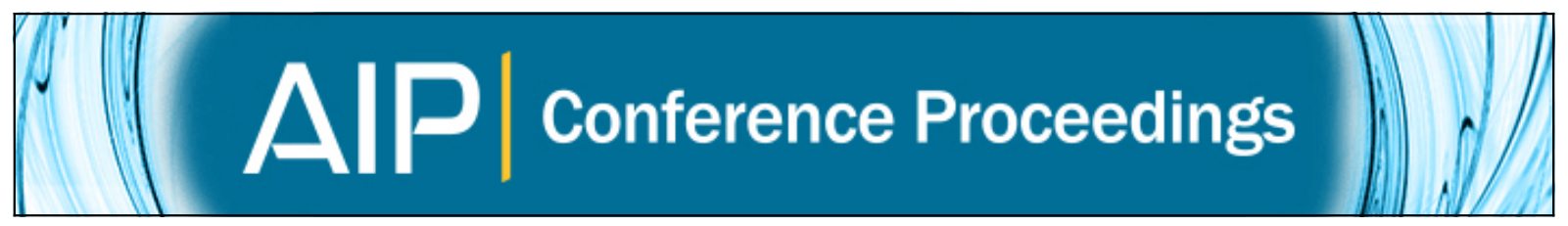

The Cryogenic Dark Matter Search (CDMS) experiment: Results, status and perspective N. Mirabolfathi, Z. Ahmed, D. S. Akerib, S. Arrenberg, C. N. Bailey, D. Balakishiyeva, L. Baudis, D. A Bauer, J. Beaty, P. L. Brink, T. Bruch, R. Bunker, B. Cabrera, D. O. Caldwell, K. Clark, J. Cooley, P. Cushman, F. DeJongh , M. R. Dragowski, L. Duong, E. Figueroa-Feliciano, J. Filippini, M. Fritts, S. R. Golwala, D. R. Grant, J. Hall, R. Hennins-Yeomans, S. Hertel, D. Homgren, L. Hsu, M. E. Huber, O. Kamaev, M. Kiveni, M. Kos, S. W. Leman, R. Mahapatra, V. Mandic, D. Moore, K. A. McCarthy, H. Nelson, R. W. Ogburn, M. Pyle, X. Qiu, E. Ramberg, W. Rau, A. Reisetter, T. Saab, B. Sadoulet, J. Sanders, R. W. Schnee, D. N. Seitz, B. Serfass, K. M. Sundqvist, G. Wang, S. Yellin, J. Yoo, and B. A. Young

Citation: AIP Conference Proceedings 1185, 623 (2009); doi: 10.1063/1.3292418

View online: http://dx.doi.org/10.1063/1.3292418

View Table of Contents: http://scitation.aip.org/content/aip/proceeding/aipcp/1185?ver=pdfcov

Published by the AIP Publishing

Articles you may be interested in

Latest Results of the Edelweiss-II Dark Matter Search Experiment

AIP Conf. Proc. 1338, 126 (2011); 10.1063/1.3579570

The Cryogenic Dark Matter Search (CDMS) : Present Status and Future AIP Conf. Proc. 1182, 260 (2009); 10.1063/1.3293795

Status of the XENON100 Dark Matter Search Experiment at LNGS

AIP Conf. Proc. 1185, 663 (2009); 10.1063/1.3292429

Status of the CRESST Dark Matter Search

AIP Conf. Proc. 1185, 631 (2009); 10.1063/1.3292420

Status of CDMS search for dark matter WIMPs

AIP Conf. Proc. 586, 107 (2001); 10.1063/1.1419540 


\title{
The Cryogenic Dark Matter Search (CDMS) experiment: Results, status and perspective
}

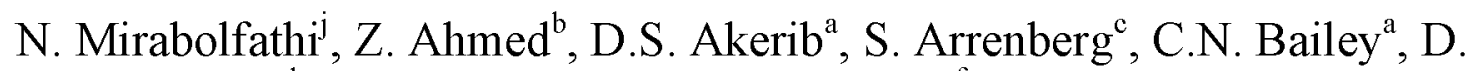

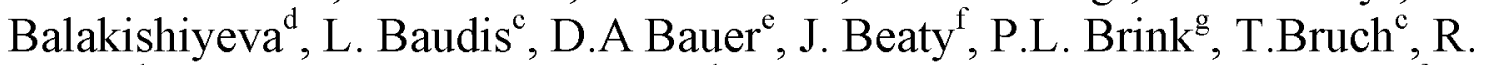
Bunker $^{\mathrm{h}}$, B. Cabrera ${ }^{\mathrm{g}}$, D.O. Caldwell ${ }^{\mathrm{h}}$, K. Clark ${ }^{\mathrm{a}}$, J. Cooley ${ }^{\mathrm{g}}$, P. Cushman ${ }^{\mathrm{f}}, \mathrm{F}$. DeJongh $^{c}$, M.R. Dragowski ${ }^{\mathrm{a}}$, L. Duong ${ }^{\mathrm{f}}$, E. Figueroa-Feliciano ${ }^{\mathrm{i}}$, J. Filippini $^{\mathrm{j}, \mathrm{b}}, \mathrm{M}$. Fritts $^{\mathrm{f}}$, S.R. Golwala ${ }^{\mathrm{b}}$, D.R. Grant ${ }^{\mathrm{a}}$, J.Hall ${ }^{\mathrm{e}}$, R. Hennins-Yeomans ${ }^{\mathrm{a}}$, S. Hertel ${ }^{\mathrm{i}}$, D. Homgren $^{\mathrm{e}}$, L. Hsu ${ }^{\mathrm{e}}$, M.E. Huber ${ }^{\mathrm{k}}$, O. Kamaev ${ }^{\mathrm{f}}$, M. Kiveni ${ }^{1}$, M. Kos ${ }^{1}$, S.W. Leman, R. Mahapatra ${ }^{\mathrm{m}}$, V. Mandic ${ }^{\mathrm{f}}$, D. Moore ${ }^{\mathrm{b}}$, K.A. McCarthy ${ }^{\mathrm{i}}$, H. Nelson ${ }^{\mathrm{h}}$, R.W.

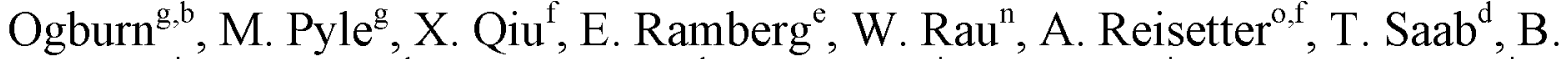
Sadoulet $^{\text {p.j }}$, J. Sanders ${ }^{h}$, R.W. Schnee ${ }^{1}$, D.N. Seitz ${ }^{j}$, B. Serfass ${ }^{j}$, K.M. Sundqvist ${ }^{j}$, G.

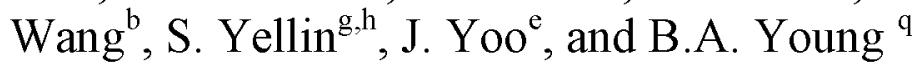

\author{
${ }^{a}$ Department of Physics, Case Western Reserve University, Cleveland, OH 44106, USA \\ ${ }^{b}$ Department of Physics, California Institute of Technology, Pasadena, CA 91125, USA \\ ${ }^{\circ}$ Physics Institute, University of Zurich, Zurich, Switzerland \\ ${ }^{d}$ Department of Physics, University of Florida, Gainesville, FL 32611, USA \\ ${ }^{\circ}$ Fermi National Accelerator Laboratory, Batavia, IL 60510, USA \\ ${ }^{f}$ School of Physics \& Astronomy, University of Minnesota, Minneapolis, MN 55455, USA \\ ${ }^{8}$ Department of Physics, Stanford University, Stanford, CA 94305, USA \\ ${ }^{h}$ Department of Physics, University of California, Santa Barbara, CA 93106, USA \\ ${ }^{i}$ Department of Physics, Massachusetts Institute of Technology, Cambridge, MA 02139, USA \\ ${ }^{j}$ Department of Physics, University of California, Berkeley, CA 94720, USA \\ ${ }^{k}$ Departments of Phys. \& Engr., University of Colorado Denver, Denver, CO 80217, USA \\ ${ }^{\prime}$ Department of Physics, Syracuse University, Syracuse, NY 13244, USA \\ ${ }^{m}$ Department of Physics, Texas A\&M University, College Station, TX 93106, USA \\ ${ }^{n}$ Department of Physics, Queen's University, Kingston, ON, Canada, K7L 3N6 \\ ${ }^{\circ}$ Department of Physics, Saint Olaf College, Northfield, MN 55057, USA \\ ${ }^{p}$ Lawrence Berkeley National Laboratory, Berkeley, CA 94720, USAq \\ ${ }^{q}$ Department of Physics, Santa Clara University, Santa Clara, CA 95053, USAThe University of California
}

\begin{abstract}
The Cryogenic Dark Matter Search experiment (CDMS) is using Phonon+Ionization detectors to search for Dark Matter in the form of Weakly Interactive Massive Particles (WIMPs). We report on new results from the operation of CDMS five "towers" at Soudan underground laboratory. With new and more massive detectors, SuperCDMS project has been started since March 2009. We report on the current status of SuperCDMS and its perspective.
\end{abstract}

Keywords: Dark Matter, WIMPs, ZIPs, Low background, CDMS.

PACS: $14.80 . \mathrm{Ly}, 95.35 .+\mathrm{d}, 95.30 . \mathrm{Cq}, 95.30 .-\mathrm{k}, 85.25 . \mathrm{Oj}, 29.40 . \mathrm{Wk}$

\section{INTRODUCTION}

Over the last decade, a variety of cosmological observations have led to the construction of a concordance model of cosmology. In this very successful model, $23 \%$ of the Universe is composed of nonbaryonic dark matter [1]. Weakly Interactive Massive Particles (WIMPs) represent a generic class of candidates for this dark matter [2]. 
The Cryogenic Dark Matter Search (CDMS) seeks to detect WIMPs via their interaction with nuclei in crystals of $\mathrm{Ge}$ or $\mathrm{Si}$ at millikelvin temperatures. CDMS uses ZIP (Z-sensitive ionization and phonon) detectors [3] to discriminate between electron-recoils (most backgrounds) and nuclear- recoils (WIMPs and neutrons) on an event-by-event basis via a simultaneous measurement of ionization and athermal phonons. Bulk and surface electron-recoils are rejected using the relative amplitudes and timings of these signals. In particular the phonon timing parameters are faster for events occurring close to the ionization electrodes (Fig. 1) where a few micron dead-layer depletes the ionization collection.
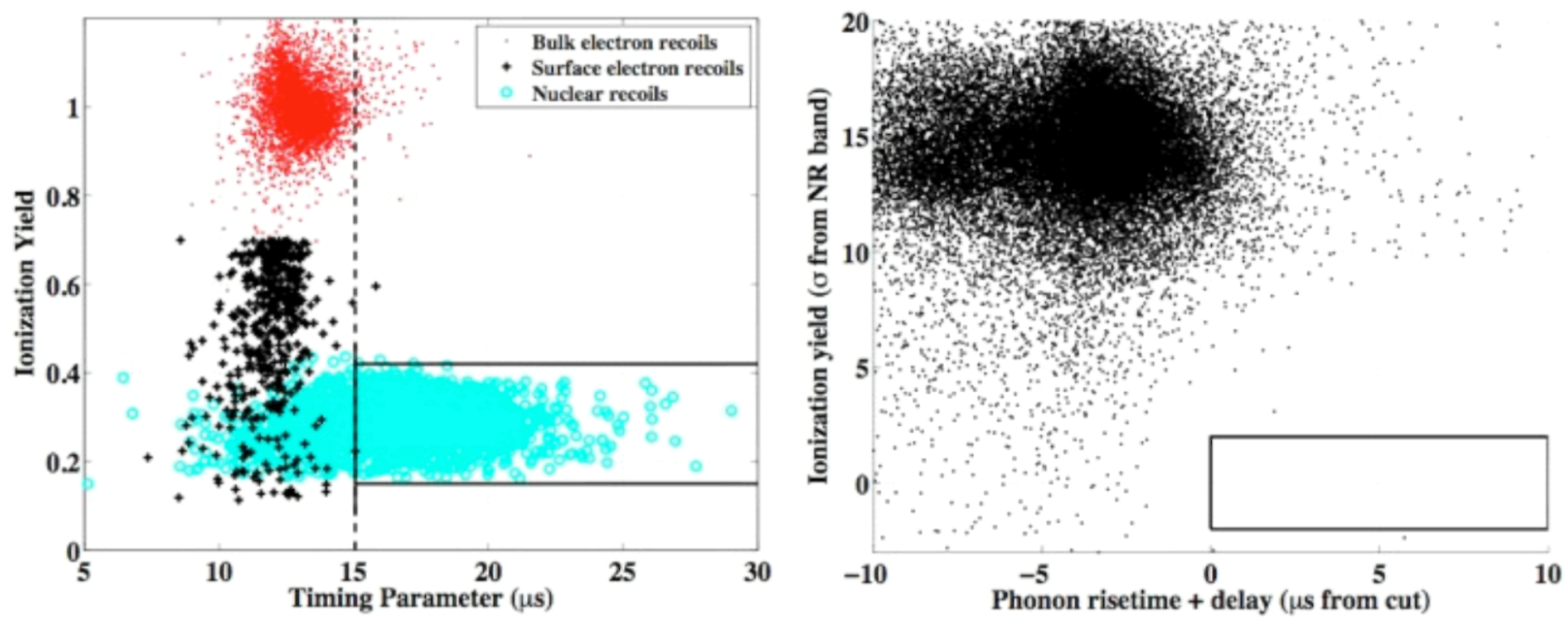

FIGURE 1. Left: Ionization yield versus phonon-timing parameter for calibration data with recoil energies $10-100 \mathrm{keV}$ in a Ge detector [5]. Three different classes of events are shown: ${ }^{133} \mathrm{Ba}$ gamma-calibration bulk (red dots) and surface (black crosses) event as well as nuclear recoils (light blue circles) from ${ }^{252} \mathrm{Cf}$. The vertical dashed line indicates the minimum timing parameter allowed for WIMP event and the box indicates the approximate signal region. Right: timing parameters and yields are scaled to overlap for all $\mathrm{Ge}$ detectors used in run 123,124 analysis. The vertical axis represents the distance in $\sigma$ from nuclear recoil band and the horizontal distance from timing cut defined based on the calibration data. No nuclear recoil candidate observed in the signal region for this data. the signal region is approximated with rectangular box.

\section{CDMSII 5 TOWER RUN RESULTS}

\section{Standard WIMP analysis}

The CDMS II project included the fabrication, testing and operation of five towers of detectors, each with six ZIP detectors. Towers $1 \& 2$ were installed at Soudan in March 2003. Data were taken with Tower 1 alone from October 2003 to January, 2004 and a second data run operating both towers was taken between March 25 and August 8, 2004. Results from the first CDMSII runs with 1 and 2 CDMSII towers were reported at the last LTD meeting [4].

In late 2004 the experiment was warmed up to install three new detector towers, the striplines to read them out, and a cryocooler to mitigate their additional heat loads and reduce helium consumption. After test runs during 2005 and early 2006 , we began taking data runs with all five towers in operation. The first two of these runs, taken between October 2006 and July 2007 , resulted in a raw exposure of $397.8 \mathrm{~kg}-\mathrm{d}, 2.5$ times the exposure of our combined previous analyses [5].

Energy calibrations were performed repeatedly during the runs using a ${ }^{133} \mathrm{Ba}$ gamma source with distinctive lines at $356 \mathrm{keV}$ and $384 \mathrm{keV}$. The agreement between data and Monte Carlo simulations and the observation of the $10.4 \mathrm{keV}$ Ga line from neutron activation of $\mathrm{Ge}$ indicated that the energy calibration was accurate and stable to within a few percent. In addition to gamma calibration data (using the ${ }^{133} \mathrm{Ba}$ source) three times per week, neutron calibration data (with an external ${ }^{252} \mathrm{Cf}$ source) were taken seven times throughout the runs to characterize nuclear recoils in the detectors.

The first analysis of the five-tower data from Soudan was based on runs taken between October 2006 and July 2007. This blind analysis incorporated more extensive data quality checks and improvements to the event reconstruction algorithm. As with previous analyses, electron recoils were distinguished 
from nuclear recoils using a combination of ionizationyield and phonon-timing cuts. These parameters were adjusted based on calibration data to maximize discovery potential by limiting expected backgrounds to less than one event in the signal region. After opening the WIMP search data, this analysis, shown in Fig. 1, revealed no candidates in $397.8 \mathrm{~kg}-\mathrm{d}$ raw exposure of the Ge detectors, consistent with a predicted background of $0.6+0.3 /-0.2$ (stat.) $+0.3 /-0.2$ (syst.) events. Over half the expected background is associated with the three detectors that are at the top or bottom of the detector stacks, and thus benefit less from self-shielding. As shown in Fig. 2, the combined data taken at Soudan result in an upper limit on the spin-independent WIMP-nucleon cross-section of $4.6 \times 10^{-44} \mathrm{~cm}^{2}$ at the $90 \%$ C.L. for a WIMP mass of 60 $\mathrm{GeV} / \mathrm{c}^{2}$, under standard assumptions about the galactic halo. These limits are a factor of $\approx 3$ stricter than our previously published results.

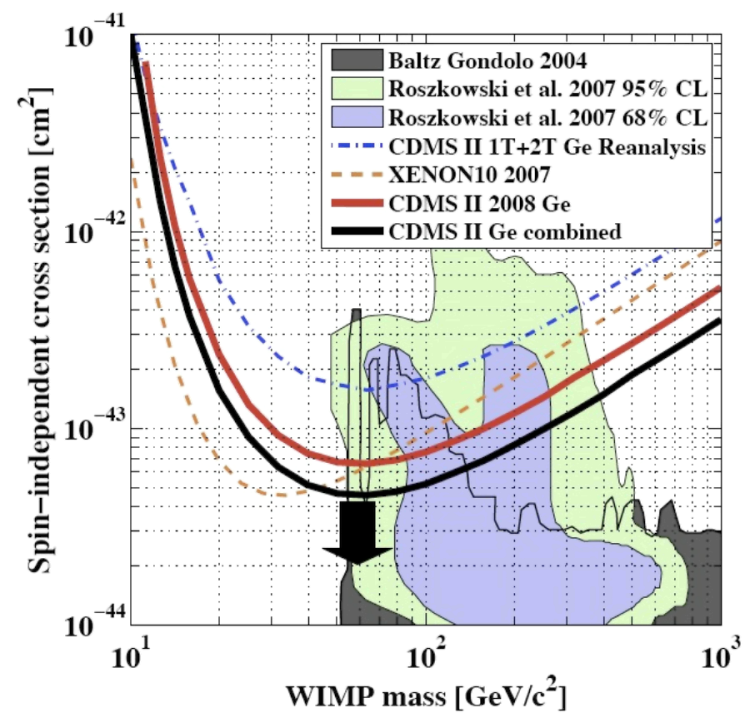

FIGURE 2. New results from CDMSII at Soudan [5], showing spin-independent WIMP-nucleon upper limits $(90 \%$ C.L) versus WIMP mass for the first 5 Tower runs and CDMS combined. Also shown on this figure represent XENON10 results (dashed) and CDMSII first two tower runs at Soudan.

All 30 detectors have been in continuous operation from July 2007 until March 2009. We accumulated further raw exposure $750 \mathrm{~kg}-\mathrm{d}$, which is almost two times greater than that used for our most recent result. Based on this projected raw exposure for CDMS II, we expect to extend our sensitivity for a WIMP-nucleon cross section down to a $90 \%$-C.L. upper limit of approximately $2.1 \times 10^{-44} \mathrm{~cm}^{2}$ at $60 \mathrm{GeV} / \mathrm{c}^{2}$ mass for our projected background of 0.5 events (without background subtraction). This expectation assumes exclusion of the detectors on the top or bottom of the detector stacks and continued modest analysis improvements leading to $2 \times$ better background rejection. The blind analysis for these data is nearly finalized and will be ready for publication toward the end of this summer.

\section{New Analysis: Low mass WIMPs and Axoinic search}

The collaboration is also extending the analysis of the CDMS II data in two new directions: the first is optimized to search for low-mass WIMPs, and the second probes for a possible solar or cosmological axion signal. For the standard WIMP limit analysis, setting the energy threshold at $10 \mathrm{keV}$ is a strict method of ensuring good electron to nuclear recoil discrimination. However, specialized analyses can push the thresholds close to the noise threshold of 0.1 $\mathrm{keV}$ for axion searches and down to $0.5 \mathrm{keV}(1 \mathrm{keV})$ for $\mathrm{Ge}(\mathrm{Si})$ for low-mass WIMP searches.

In particular, we performed analysis of the lowenergy electron-recoil spectrum from the CDMS II experiment using data with an exposure of $443.2 \mathrm{~kg}$ days [7]. The analysis provides details on the observed counting rate and possible background sources in the energy range of $2-8.5 \mathrm{keV}$. There is no significant excess in the counting rate above background. In the framework of a decaying dark matter particle we directly compare the $90 \%$ confidence level upper limits on an excess rate above background of 0.246 $\mathrm{cpd} / \mathrm{kg}$ at $3.15 \mathrm{keV}$ to the total signal rate observed by DAMA, which is greater by $8.9 \%$. In the absence of any specific particle physics model to provide the scaling in cross section between $\mathrm{NaI}$ and $\mathrm{Ge}$, we assume a $Z^{2}$ scaling. With this assumption the observed rate in DAMA differs from the upper limit in CDMS by $6.8 \sigma$. Under the conservative assumption that the modulation amplitude is $6 \%$ of the total rate we obtain upper limits on the modulation amplitude about a factor of $>2$ less than observed by DAMA constraining some possible interpretations of this modulation [7].

An energy threshold of $2 \mathrm{keV}$ for electron recoil events allows a search for possible solar axion conversion into photons or local Galactic axion conversion into electrons in the germanium crystal detectors. CDMS solar axion search sets an upper limit on the Primakov coupling $g_{a y y}$ of $2.4 \times 10^{-9} \mathrm{GeV}^{-1}$ at the $95 \%$ confidence level for an axion mass less than 0.1 $\mathrm{keV} / \mathrm{c}^{2}$. This limit benefits from the first precise measurement of the absolute crystal plane orientations in this type of experiment. The Galactic axion search analysis sets a world-leading experimental upper limit on the axio-electric coupling $g_{a \bar{e} e}$ of $1.4 \times 10^{-12} \mathrm{GeV}^{-1}$ at the $90 \%$ confidence level for an axion mass of 2.5 
$\mathrm{keV} / \mathrm{c}^{2}$. This analysis excludes an interpretation of the DAMA annual modulation result in terms of Galactic axion interactions for axion masses above $1.4 \mathrm{keV} / \mathrm{c} 2$ [6].

\section{SuperCDMS}

SuperCMDS is the extension to CDMS with the goal to increase its exposure by further factor of 10100. Based on successful ZIP technology The first phase of SuperCDMS also called "SuperCDMS Soudan ", consists of operating $15 \mathrm{~kg}$ of 1 inch thick ZIP detectors [8]. For this phase of the project, we will use the same experimental setup as CDMSII at Soudan underground laboratory. Considering our current background estimates and experimentally proven discrimination improvements with SuprCDMS new detectors, we expect to reach sensitivity goal of $5 \times 10^{-45} \mathrm{~cm}^{2}$ at a very low risk: A factor of $x \sim 10$ below our currently published results and $x \sim 5$ below CDMSII expected final sensitivity. In particular, Our new 1 inch detectors are well characterized at CDMS dilution fridge test facilities and shown to have the discrimination capabilities required for the SuperCMDS Soudan projected sensitivities [8].

SuperCMDS is currently funded for fabrication and operation of two Supertowers (each stacking five 1 inch Ge detectors with two top and bottom $1 \mathrm{~cm}$ veto endcaps as shown in figure 3) and is seeking for funding to fabricate three more SuperTowers and operate at its full capacity until the end of 2011.

Since March 2009 the first SuperCMDS SuperTower has been successfully installed and cooled to $<40 \mathrm{mK}$ and is currently in process of phonon sensor fine tuning and commissioning.

In order to reach sensitivities below $10^{-45} \mathrm{~cm}^{2}$ CDMS is proposing SuperCDMS SNOLAB to increase the total detector mass to $100 \mathrm{~kg}$ Ge and the sensitivity reach of $3.1 \times 10^{-46} \mathrm{~cm}^{2}$. CDMS is currently investigating several enhanced detector readout schemes to improve its background per kilogram to the levels required by SuperCDMS SNOLAB project [9][10]. Also to mitigate the risk of contamination from Muon induced neutron background we propose this new project to be installed in a new deeper facility at SNOLAB.

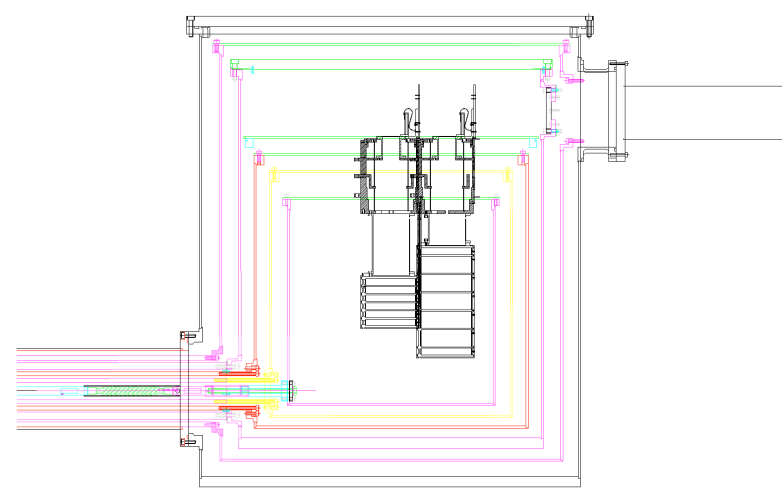

FIGURE 3. Comparison between SuperCDMS 1 inch thick ZIP Supertower and CDMS $1 \mathrm{~cm}$ thick ZIP tower in the Soudan icebox. A SuperTower cosnist of 5, 1 inch Ge ZIPs with two $1 \mathrm{~cm}$ Ge veto detectors at both ends.

\section{ACKNOWLEDGMENTS}

This work is supported in part by the National Science Foundation (Grant Nos. AST9978911,PHY-0542066,PHY-0503729,PHY-0503629, PHY-0503641, PHY-0504224, PHY-0705052, PHY0801536, PHY-0801708, PHY-0801712 and PHY0802575), by the Department of Energy (Contracts DE-AC03-76SF00098, DE-FG02-91ER40688, DEFG02-92ER40701, DE-FG03-90ER40569, and DEFG03-91ER40618), by the Swiss National Foundation (SNF Grant No. 20-118119), and by NSERC Canada (Grant SAPIN 341314-07).

\section{REFERENCES}

1. D.N. Spergel et al., Astrophys. J. Suppl. 148, 175 (2003).

2. G. Jungman et al., Phys. Rep. 267, 195 (1996).

3. T. Saab, et al., Nucl. Instr. and Meth. A 444 (2000) 300

4. Z. Ahmed et al. J Low Temp Phys (2008) 151: 800-805.

5. Z. Ahmed et al. Phys. Rev. Lett. 102, 011301 (2009).

6. Z. Ahmed et al. arXiv:0902.4693v1

7. Z. Ahemd et al. arXiv:0907.1438

8. Z. Ahmed et al. this proceeding

9. M. Pyle et al. this proceeding

10. M. Daal et al. this proceeding 